\title{
EVEN AND ODD ENTIRE FUNCTIONS
}

\author{
A. F. BEARDON \\ (Received 6 July 2001; revised 11 October 2001) \\ Communicated by P. C. Fenton
}

\begin{abstract}
We examine what can be said about a polynomial $p$ and an entire function $f$ given that $p \circ f$ is an even, or an odd, function.

2000 Mathematics subject classification: primary 30B 10, 30D05, 30D20.

Keywords and phrases: entire functions, even functions, uniformization.
\end{abstract}

\section{Introduction}

An entire function $f$ is an analytic map of the complex plane $\mathbb{C}$ into itself, and any entire function $f$ can be expressed uniquely as the sum $f=f_{E}+f_{O}$ of an even entire function $f_{E}$ and an odd entire function $f_{O}$ in the usual way. The composition of two maps $f$ and $g$ is denoted by $f \circ g$. We shall use these notations throughout the paper.

In [1, page 228] the author asks for a characterization of polynomials $p$ and entire functions $f$ such that $p \circ f$ is even, and remarks that the existence of an algebraic relation between $f_{E}$ and $f_{O}$ is a necessary, but not a sufficient, condition for $p \circ f$ to be even. We provide a simple characterization in Section 2, and this shows quite clearly why the existence of an algebraic relation is necessary but not sufficient. In [1] and [2], the authors discuss criteria that imply that if $f \circ g$ is even, where $f$ and $g$ are entire, then $f$ or $g$ is even. We suggest that this may not be the right question to ask, and we develop this idea in Section 3. Finally, in Section 4 we show that [1, Example 3.1], given to illustrate [1, Theorem 3.1], is essentially the only possible example that could have been given, and we place this example in a more general context.

(C) 2003 Australian Mathematical Society $1446-7887 / 03 \$ A 2.00+0.00$ 


\section{The characterization}

Given a polynomial $p$ in one complex variable, we define polynomials $\Phi_{p}$ and $\Psi_{p}$ in two variables by

$$
\Phi_{p}(u, v)=p(u+v)-p(u-v), \quad \Psi_{p}(u, v)=p(u+v)+p(u-v)
$$

THEOREM 2.1. Let $p$ be a nonconstant polynomial, and let $f$ be a nonconstant entire function. Then

(a) $p \circ f$ is even if and only if for all $z, \Phi_{p}\left(f_{E}(z), f_{o}(z)\right)=0$;

(b) $p \circ f$ is odd if and only if for all $z, \Psi_{p}\left(f_{E}(z), f_{o}(z)\right)=0$.

ProOF. This is trivial, for obviously

$$
\begin{aligned}
& \Phi_{p}\left(f_{E}(z), f_{O}(z)\right)=p(f(z))-p(f(-z)), \\
& \Psi_{p}\left(f_{E}(z), f_{O}(z)\right)=p(f(z))+p(f(-z)) .
\end{aligned}
$$

This shows that if $p \circ f$ is even then $f_{E}$ and $f_{O}$ are algebraically related but, of course, only the algebraic relation $\Phi_{p}=0$ (or a relation which has $\Phi_{p}$ as a factor) will guarantee that $p \circ f$ is even. The corresponding statement holds for odd functions and the polynomial $\Psi_{p}$.

Notice that Theorem 2.1 enables one to characterize, for a given polynomial $p$, all entire functions $f$ for which $p \circ f$ is even, and an example will suffice to illustrate this. Let $p(z)=z^{3}+z$. Then $\Phi_{p}(u, v)=2 v\left(3 u^{2}+v^{2}+1\right)$ so that $p$ of is even if and only if either $f_{o}=0$ (so that $f$ is even), or $3 f_{E}^{2}+f_{o}^{2}+1=0$. Of course, for algebraic reasons $\Phi_{p}(u, v)$ will always have a factor $v$, and this corresponds to the analytic fact that if $f$ is even, then so is $p \circ f$ for every $p$.

The example $f(z)=\sinh z+1$ and $p(z)=z^{2}-2 z$ shows that $p \circ f$ may be even while $p$ and $f$ are not. Here, $p \circ f$ is even because $\Phi_{p}(u, v)=4 v(u-1)$ and $f_{E}(z)=1$ for all $z$. The example $f(z)=\sin z+1$ and $p(z)=z-1$ shows that $p \circ f$ may be odd while $p$ and $f$ are not. In this case, $p \circ f$ is odd because $\Psi_{p}(u, v)=2(u-1)$ and $f_{E}(z)=1$ for all $z$.

\section{Some remarks}

In [2] the authors ask whether $f \circ g$ being even (where $f$ and $g$ are entire) implies that either $f$ or $g$ is even, and they then give the example $f(z)=(z-1)^{2}$ and $g(z)=z+1$ to show this is not so. We suggest, however, that this may not be the correct question to ask as the given data, namely the single function $f \circ g$, does not 
determine $f$ and $g$ uniquely. This suggests the following question: if a composition $F$ of two entire functions is even, can we express $F$ as a nontrivial composition of two functions at least one of which is even? Now this question has a trivial answer as any even entire function is an entire function of $z^{2}$; so, once again, we have to modify the question. Perhaps the 'right' question is: if $f$ and $g$ are entire functions, and iff $\circ \mathrm{g}$ is even, is there a linear polynomial $t$ such that either $f \circ t$ or $t^{-1} \circ g$ is even? The choice of the class of linear polynomials here is natural as these are the automorphisms of $\mathbb{C}$. Note that the counterexample in [1] (and given above) is not a counterexample to the modified question for (with the same $f$ and $g$ as above) $f \circ g=(f \circ t) \circ\left(t^{-1} \circ g\right)$, and $f \circ t$ is even when $t(z)=z+1$.

The authors of [1] and [2] also make frequent use of an assumption $f(0)=0$ or $g(0)=0$. For example, in [2] they remark that 'the problem becomes more interesting if one assumes that $g(0)=0$ ' and they then prove that if $p$ and $q$ are polynomials with $q(0)=0$ and $p \circ q$ even, then $p$ or $q$ is even [2, Theorem 1]. Here, the assumption $q(0)=0$ seems arbitrary, but it appears naturally in this modified setting for now their Theorem 1 reads as follows: if $p \circ q$ is even, then there is a linear polynomial $t$ such that $p \circ$ or $^{-1} \circ q$ is even. Thus their Theorem 1 answers the question posed above when $f$ and $g$ are polynomials.

\section{An example}

Theorem 3.1 in [1] states that: if $f$ is entire, and if $f_{E}^{2}+f_{O}^{2}=1$, then $p \circ f$ is even, where $p(z)=z^{4}-2 z^{2}$, and this is then illustrated by the example $f(z)=\cos z+\sin z$. We shall now show that this is essentially the only example that could have been given here. First, if $p(z)=z^{4}-2 z^{2}$ then $\Phi_{p}(u, v)$ has a factor $u^{2}+v^{2}-1$, and this is why $p \circ f$ is even when $f_{E}^{2}+f_{O}^{2}=1$.

Suppose now that the two entire functions $s$ and $t$ satisfy $s^{2}+t^{2}=1$ throughout $\mathbb{C}$. Then $(s+i t)(s-i t)=1$, so that neither factor vanishes, and this means that there is an entire function $h$ such that $s+i t=e^{i h}$ and $s-i t=e^{-i h}$. Thus $s(z)=\cos h(z)$ and $t(z)=\sin h(z)$. Now suppose that, in addition, $s$ is even and $t$ is odd. Then as

$$
\begin{aligned}
& \sin h_{E}(z) \sin h_{O}(z)=-s_{O}(z)=0, \\
& \sin h_{E}(z) \cos h_{O}(z)=t_{E}(z)=0,
\end{aligned}
$$

we see that $\sin h_{E}(z)=0$ for all $z$; thus $h_{E}(z)=k \pi$ for some integer $k$. It follows from this discussion that if $f$ is entire, and if $f_{E}^{2}+f_{O}^{2}=1$ then, by taking $f=s+t$, we see that

$$
f_{E}(z)=s(z)=(-1)^{k} \cos h_{O}(z), \quad f_{O}(z)=t(z)=(-1)^{k} \sin h_{O}(z) .
$$


There are many algebraic relations for which a similar result holds, and the explanation lies in the theory of uniformization of algebraic curves, and in the fact that an algebraic relation or, equivalently, an algebraic curve, is essentially a compact Riemann surface. In the example above, the algebraic curve is given by $u^{2}+v^{2}-1=0$, the corresponding Riemann surface is the Riemann sphere, and the algebraic curve is uniformized by the pair of functions $\sin z$ and $\cos z$. Not every algebraic curve arises in this way, for Picard proved that if an algebraic curve can be uniformized by a pair of entire functions (in our case, by $f_{E}$ and $f_{O}$ ), then the corresponding Riemann surface is topologically a sphere. We end with an example to illustrate this idea, and this example will also serve as another application of Theorem 2.1.

EXAMPLE 4.1. Let $P(u, v)=(8 u+1) u^{2}-9 v^{2}$, and suppose that $s$ and $t$ are nonconstant entire functions such that $P(s, t)=0$, where $s$ is even and $t$ is odd. We shall show that there is an odd entire function $g$ such that

$$
s(z)=\frac{1}{8}\left(4 g(z)^{2}-1\right), \quad t(z)=\frac{1}{12}\left(g(z)\left[4 g(z)^{2}-1\right]\right) .
$$

PROOF. We begin with the graph of $P$, namely

$$
\mathbf{P}=\{(u, v) \in \mathbb{C} \times \mathbb{C}: P(u, v)=0\},
$$

and this is embedded in projective space $\mathbb{P}_{2}$ in the usual way by using homogeneous co-ordinates. The projective model of this graph is the set $\mathbf{P}^{*}$ of projective points $(u, v, w)$ for which $(8 u+w) u^{2}+9 v^{2} w=0$, and this meets the line at infinity $(w=0)$ at the single projective point $(0,1,0)$. Thus $\mathbf{P}^{\#}$ is obtained from $\mathbf{P}$ (or, strictly, a projective copy of $\mathbf{P})$ by adding the single projective point $(0,1,0)$, and $\mathbf{P}^{\#}$ is conformally equivalent to the extended complex plane $\mathbb{C}_{\infty}$ (for, as we shall see below, $\mathbf{P}$ is uniformized by two polynomials $p$ and $q$ ). We shall not need these facts, but they underpin much of our argument.

Now let

$$
p(z)=z(z+1) / 2, \quad q(z)=z(z+1)(2 z+1) / 6,
$$

so that $3 q(z)=(2 z+1) p(z)$. Then

$$
P(p(z), q(z))=p(z)^{2}\left[8 p(z)+1-(2 z+1)^{2}\right]=0,
$$

so that the map $\theta: z \mapsto(p(z), q(z))$ maps $\mathbb{C}$ into $\mathbf{P}$. In fact, $\theta$ is a bijection of $\mathbb{C} \backslash\{0,-1\}$ onto $\mathbf{P} \backslash\left\{(0,0\}\right.$ because the point $\left(u_{0}, v_{0}\right)$ on $\mathbf{P}$, where $u_{0} \neq 0$, is the $\theta$-image of exactly one point in $\mathbb{C}$, namely $\left(3 v_{0}-u_{0}\right) /\left(2 u_{0}\right)$.

Now suppose that $s$ and $t$ are entire functions with $P(s, t)=0, s$ even and $t$ odd, and let $\mu: \mathbb{C} \rightarrow \mathbf{P}$ be given by $\mu(z)=(s(z), t(z))$. Then the map $h=\theta^{-1} \circ \mu$ 
is defined and analytic on the set $\mathbb{C} \backslash Z$, where $Z$ is the set of points $z$ in $\mathbb{C}$ where $s(z)=t(z)=0$. Clearly, the points of $Z$ are isolated, and if $z$ is near a point of $Z$, then $h(z)$ is in some neighbourhood of 0 or -1 ; thus each point of $Z$ is a removable singularity of $h$, and so $h$ is entire.

Next, $\theta \circ h=\mu$ so that $p \circ h=s$. By assumption, $s$ is even, so, from Theorem 2.1, $\Phi_{p}\left(h_{E}, h_{o}\right)=0$. Now as $p(z)=z(z+1) / 2$, we see that $\Phi_{p}(u, v)=v(2 u+1)$, so that $h$ is even or $2 h_{E}+1=0$. As $q \circ h=t, h$ is not even (else $t$ is even and odd and hence identically zero); thus $2 h_{E}+1=0$ and this means that $h(z)+h(-z)=-1$. We now let $g=h+1 / 2$; then $g$ is odd, and $s=p \circ h=\left(4 g^{2}-1\right) / 8$ and similarly for $t$. The proof is complete.

\section{References}

[1] A. L. Horwitz, 'Even compositions of entire functions and related matters', J. Austral. Math. Soc. (Ser. A) 63 (1997), 225-237.

[2] A. L. Horwitz and L. A. Rubel, 'When is the composition of two power series even?', J. Austral. Math. Soc. (Ser. A) 56 (1994), 415-420.

Centre for Mathematical Sciences

University of Cambridge

Wilberforce Road

Cambridge CB3 0WB

England

e-mail: afb@dpmms.cam.ac.uk 
